\title{
Rainer Wolf Das Bundesverfassungsgericht - Hüter der Umwelt?
}

\author{
1. Zwei altemative Konzepte politischer Steuerung und rechelicher Kontrolle: \\ Umweltrecht zwischen Ergebnisorientierung und Prozeduralisierung
}

Umweltschutz findet heute zumindest in rhetorischen Beschwichtigungsformeln wals Schicksalsaufgabe des modernen Staates ${ }^{2}$ allgemeine Aufmerksamkeic. Gleichwolsl fehlt eine verfassungsrechtliche Justierung eines ökologischen Überlebensprogrammes im Grundgesetz. De lege ferenda scheint ein Grundrecht auf menschenwürdige Umwelt nach den anfänglichen Ambirionen der sozial-liberalen Koalition in den írühen siebziger Jahren mitclerweile ohne Chancen zu sein. ${ }^{2}$ Hingegen ist die Einfügung einer neuen Staatszielbescimmung "Umweltschurz inzwischen fast schon zum verfassungspolitischen Markenzeichen konservativer Umweltpolitik avanciert, dem die Opposicion kaum mehr entgegenzuserzen vermag als den Anspruch, diese Position bereits von Anfang an vertreten zu haben. Die dahinterscehende politische Intention ist leicht auszumachen. Während ein Umweltgrundrecht eine von den Gerichsen nur schwer zu bewältigende Anspruchslawine auf bisher versäumte Maßnahmen zum Schucz der Umwelt auslösen müßte, verspricht eine für das Systcm des Individualrechrsschutzes weit weniger folgenreiche Staatszielbestimmung vor allem einen legitimatorischen Effekt.'

Im Zusammenhang mit diesen verfassungsinnovativen Aktivitäten der politischen Parteien erscheint die hier zu konstarierende Zurückhalcung des Bundesverfassungsgeriches bemerkenswert. Is es doch seir mehreren Jahren in die umweltpolitische Kontroverse und ihre rechtsförmigen Muster der Konfliktbearbeicung involvier, ohne die Normierung eines Umweltgrundrcchts oder einer umweltschüzzenden Staatszielbestimmung für verfassungsrechtlich erforderlich zu exklären. ${ }^{4}$ Vielmehr entwickelt es seine Rechtsprechung zum Umweltschucz aus seiner allgemeinen Verfassungsrechrsdogmatik heraus.

$\mathrm{Da}$ der Verfassungsgeserzgeber in Ansatz des Bundesverfassungsgeriches für die

1 R. Breuer, Umwejtschutzrecht, in: v. Münch (Hrsg.), Besandcres Verwaltungsneche, 6. Aufl. Bcr. lin-New York i $982,5.644$.

$2 \mathrm{Vgl}$. Umiveliprogramm der Bundesregierung von 1971, BT-Drs VI/2710; Die Freiburger Thesen der Liberalen, Reinbek 1972, S. 109 ff; A rbeitsgemeinschaft Sozjaldemokracischer Juristen, ZRP 72, 76; Rat von Sachverständigen für Umweltíragen, Umweltgutachtcn 1976, Rdn. 634 ff.; xurn derzejtigen Meinungsscund vgl. Sendler, UPR 81, 3; Benda, UPR 82, 244; Breucr, 2. 2. O.

3. Wie die politische Wissenschaft hinreichend belege hat, ist die Präferenz für osymbolic use of politics. (vgl. dazu Murray Edelman, Policik als Rituai. Die symbolischen Funktionen scastlicher Institutionen und polidischen Handelns. Frantefurt 5976, S. 22 ff.) immer dann zu beobachten, wenn reale Verinderungen auf starken Widerseand seoßen ruurden. Dies istauch in bezug aul die neueste Diskussion über dic Änderung von Landesveríassungen und des Grundgesetzes festzuscellen (vgl, dazu Der Spiegel, 28/1984). Wolke man wirklich dic recheliche Konuralldiche crhohen, ware dic Einfuilirung der Verbandsklage wolli das iagglichere Mincl als eine Verfassungsanderung.

4 Vgl. dazu Sendler, a. a. O. 
rechrlichen Grundlagen der Umweltpolitik niche gefordert ist, läßı den Schluß zu, die vom Bundesverfassungsgericht ausgeübten Funktionen der politischen Steuerung und Kontrolie im Integral der suprema porestas' scien mictlerweilc so unangreifbar, da $ß$ sie von einem neuen verfassungsreformatorischen $V_{\text {errechrlichungs- }}$ schub kcinen wesentlichen politischen Zugewinn erwarten könnten. Somit dokumentiert gerade die ohne formelle verfassungsmäßige Institutionalisierung des Umwelrschurzes ausgeübre Judikatur des Bundesverfassungsgerichts den Spielraum seiner politischen Gestaltungsmacht.

Vier Entscheidungen haben hicr besondere Beachtung verdiemt

(I) Der "Kalkar-Beschluß zurn "Schnellen Brürer vom 8.8. $197^{8}$ (BVerfGE 49 , 89 lf.)

(2) Der "Mülheim-Kärlich-Beschluß vom 20. 12. 1979 (BVerfGE 53, 30 ff.)

(3) Der „Fluglärm-Beschluß " zum Flughafen Düsseldorf-Lohausen vom I 4. 1. 1981 (BVerfGE $56,54 \mathrm{ff}$.)

(4) Der "Naßauskiesungs-Beschluß« zum Wasserhaushaltsrecht vom 15. 7. 198 t (BVerfGE s $8,300 \mathrm{ff}$.)

Im folgenden wird untersuchr, auf welche verfassungsrechclichen Konflikckonstellationen sich die Entscheidungen des Bundesverfassungsgeriches beziehen und mir welchen normativen Leitbildern die Lösungsversuche der Rechrsprechung operieren. Obwohl der Umweltschutz im Zentrum der polisischen Aufmerksamkeic liegt, zeige bercits die prozessuale Ausgangsposition - Verfassungsbeschwerden und Vorlagebeschlüsse -, daß hier vor dem Verfassungsgericht kein Konflikt zwischen den dominierenden Macheträgern des politischen Systems ausgetragen wird. Vielmehr scheint das Bundesverfassungsgericht cinmal mehr als "Superrevisionsinstanz" angerufen zu werden, um Konsistenzprobleme der Verrechtlichung des Umweltschutzes und die Akzeptanzprobleme bereits justitialisiercer Konfliktentscheidungen zu lösen. Damir wird deutlich, wo die politische Konfrontationslinie verläuft, über die das Gericht zu befinden har. Es geht um die Kritik, die von der Peripherie des politischen Systems durch Bürger, die mit den tradierten Lösungsmustern der Programmentwicklung und Implemenzacion des Umweluschuczes niche mebr zufrieden sind, arcikuliert wird, während die Grundstrukturen der Umweltpolitik im Zenzrum der politischen Herrschaft uncer Parteien, Regierungen und Behörden weichin unbestritten sind.

Angerufen zur Kompensation der selektiven Problem- und Interessenberücksichtigungsmuster der dominierenden Machtkoalicion kann sich das Bundesverfassungsgericht an zwci begrifflich zu unterscheidenden Kontrollmodellen orientieren. Den Ansprüchen der deurschen Rechesstaatsentwicklung entsprechend liegt ein Schwerpunke der justizförmigen Kontrolle politisch-administrativen Handelns in materiellrechtlichen Richtigkeitserwägungen, die sich primär auf das Ergebnis der jeweiligen Entscheidungen bezichen'. Dem entspricht die Lehre zu den im Umweltrecht so zentralen unbestimmeen Rechtsbegriffen, nach der die juristische Operationalisierung von gesetzlichen Blankettiormeln wie "Gefahr" ( $\$$; Nr. , BImSchG), „Vorsorgea ( $\$ ; \mathrm{Nr}, 2 \mathrm{BImSchG}$ und $\$ 7 \mathrm{II}$ Nr. $3 \mathrm{ArG}$ ), nallgcmcin anerkannte Regeln der

s Vgl. zur politikwissenschaftlichen Analyse Otwin Massing, Das Bundesveriassungsgeriche als lnstrument sozialer Kontrolle, in: Mchdi Tohidipur (Hrsg.), Verfassung, Verlassungsgcrichtsbarkeit, Poliuk, Frankluramin $1976,5.10(381$.$) .$

6 Damit ist juloch das Arsenal der umweltelevanten Entscheidungen des Gerichts noch nicht erschopfi. Neben den späteren Judikaten zum a dings primär im enteignungs- und planungsrechulichen Kontext ergangenen Enkrcheidungen zur $\bowtie H_{2} m$. burger U-Bahn * (BVerfGE 45. 297 If.) und zur n Bad Dürkheimer Gondelbahn * (BVerfGE 56,248 If, ) rvichtige umweltpolitische Problemsicllungen zugrundc.

7 Vgl. dazu F. W. Scharpf, Dic politischen Kosten des Rerhisstaaes, Tübingen 1970 
Technik ( $\$$ 7a WHG), „Stand der Technik ( $\$$ S Nr. z BImSchG) oder „Stand von Wissenschaft und Technik $(\$ 7$ II Nr. 3 AtG) nur eine rechtlich zutreffende Entscheidung ermöglicht. Dieser essentialistische Rechtsbegriff verlangt nach einer aus dem Recht definitiv ableitbaren Entscheidung über die Fragen:

- Wie sicher isı sicher genug?

- Welche umweltpolitischen Grenzwertbestimmungen ergeben sich aus dem Schurzzweck des Geselzes?

- Welche umweltgefährdenden Handlungen sind grundsä̌clich zu unterbinden?

Dem entspricht cin verbreiteter Politikbegriff, der gleichfalls allein auf Ynhalte fixiert ist. Wie unpolitisch solche Auffassungen sind, zeigt sich darin, daß sich hier die Sichtweisen von ferischisierten ökonomischen und ökologischen Positionen treffen. Für die einen ist die Umweltpolitik durch die Kalküle volkswirtschafrlicher Racionalitätsberechmungen bestimmbar, für die anderen gibı die Integrität ökologischer Kreisläufe die unabänderliche Marschzahl für die Umweltpolitik vor. Zu Recht konstaciert ein kricischer Beobachter umweltpolitischer Grenzwertkonzepte für beide kontroversen Standpunkte ein gchcimes Einverständnis: "for both of them, the important thing is outcome, not process; both are interested in the decisions that are made, not how they are mader 8 .

Damit ist das alternative Kontrollmodell bereits angedeutet: „rationality of process, not of outcome «?. Eine prozeßorientierte Rechtmäßigkeitskontrolle hat demgemäß daraú abzustellen,

- auf welcher Ebene der horizonulen Gewaltenteilung die umweltpolitıschen Lcitentscheidungen fallen müssen,

- in welchen Formen die Programmentwicklung statczufinden hat,

- welche Verf́ahren und Becciligungen die administrativen Implementacionsprozesse zu beachten haben und welche Abwagungsvorgänge den Einzelentscheidungen zugrunde liegen müssen.

Die eben skizzierte Differenzierung zwischen ergebnis- und prozcßorientierten RechtmäBigkeitskriterien ist Juristcn aus dem Planungsrecht bekannt, wo zwischen Planen als Vorgang und dem Plan als Produkt dieses Vorganges unterschieden wird ${ }^{10}$. Sie wird dort als komplementäre Anlage von richterlichen Prüfungsdimensionen verstanden. Zumindest für die hier zu behandelnde Ebene der verfassungsrechtlichen Juscierung der Umweltpolitik ergeben sich aber auch unterschiedliche Kontrollfunkrionen. Während eine ergebnisorientierte Judikatur dem Geseczgeber den Inhalt der Umweltpolitik vorgibr bzw. den von ihm verfolgten Kurs posituv legitimiert, readressiert eine prozeßorienrierrc Kritik umweltpolitischer Entscheidungen die Problemlösung lediglich erneut an die primär zuständigen politischadministrativen Instanzen mit der Maßgabe der form- und funktionsgerechten Wahrrehmung ihrer Aufgaben. Suggeriert die erste Lösung leicht die verfassungsrechtlich zu gewährleistende Garantie eines ökologischen »juste milieu «", so präreriert die zweite Lösung in bezug auf die Policikinhalte dic Deklaracion des njudicalself restraint " und der »political question « und setzs statt dessen auf die korrektive Kraft von formal-rationalen Entscheidungsbindungen, die die Theorie demokracischer und rechesstaatlicher Verfahren postuliert ${ }^{12}$.

8 G. Majone, ZTU 82, jog $(306)$.

9 A. A. O., S. 307 .

10 Vgl. dazu BVerwGE $41,67(71): 45,309$ (312) .Floatglas。 und jüngst Ladcur, UPR $\$_{4}$, I ff. mil der Betonung prozeduraler Abrvigungskritenen.

"Vgl, zu ähnlichen Tendenzen $d e s$. Wirschafesverfassungsrechus die hier adapuere Kritik von H. Elumke (Wirschali uod Verfassung, Karlsruhe 1961, S. 57 fI.).

12 Auf den rechestheorerischen Hintengrund dieser Überlegungen kann hier nur kurz bungewiesen werden: Scit einiger Zeit wird dic bereits von Mas Weber dingnostuziere Krise des formalen Rechis angesiches 
Der „Kalkar-Entscheidung ( $B V \operatorname{cr}\left(G E{ }_{49}, 89\right.$ ) liege ein Vorlagebeschluß des OVG Münster gemäß Art. 100 I GG zugrunde (NJW 78, 439), der auf prozeßorientierten Erwägungen beruht. Haste das OVG Münster unter Hinweis auf die Wesentlichkeitstheorie's die Entscheidung des Parlaments über den Einstieg in eine so folgenreiche Technologie wie die des "Schnellen Brürcrs für verfassungsrechelich unabdingbar gehalten, so zielt der Beschluß des Bundesverfassungsgerichts in Gegensatz dazu gerade auf die Thematisierung ergebnisorientierter Erwägungen. Im gleichen Umfang wie hier der ndynamische Grundrechtsschurz ( $S$, 137 ) durch eine Risikoabschäızung nach dem Standard npraktischer Vernunfr" (S. 143) materiell als gewährleistbar erachret wird, verlieren die prozeduralen Bedenken des OVG Münster an Gewicht, nur durch eine förmliche Parlamentsentscheidung könne die Substanz des demokratischen Rechrsstaates bei der Kontrolle moderner Technologieentwicklung erhalten bleiben.

Damit erhält der in der Wesentlichkeitscheorie dahingehend präzisierte Grundsatz des allgemeinen Gesetzesvorbehalts, der Gesetzgeber müsse in den von ihm erlassenen Normen das Wesentliche eines Politikbereiches selbst festlegen und dürfe dies niche der Ausgestaltung durch die Verwaltung überlassen, für das Recht des Umwelsschutzes und der rechnischen Sicherheit einen grundsärzlich weniger anspruchsvollen Rang ais etwa im Schulrechr. Hier ist die Einführung der obligatorischen Förderscufe's, die Regelung des Schulabschlusses's, die Einrichrung der reformierten Oberstufe ${ }^{16}$ und sogar des Sexualkundeunterrichts' 17 für parlamentspflichtig erachtet worden. Die 1959 für die Genehmigung von Kernenergieanlagen formulierce geseczliche Vorausseczung, die nach dem »Stand von Wissenschaft und Technik « erforderliche Vorsorge gegen die Gefahren ionisierender Strahlen müsse gewährleistet sein ( $\$>$ II Nr. 3 AtG), reicht nach Ansicht des Zweiten Senates aus, um das Wesentiche der durch die Kernenergie verursachten Gefahrendimensionen als parlamentsförmig geregelt zu sehen (S. I29 ff.). Das damals bereits $20 \mathrm{Jahre}$ alte Gesetz vermag so die Abfolge ganze Reaktorbaulinien und den Paradigmenwandel in den «Sicherheirsphilosophien« ohne normativen Schaden an seiner Regelungsqualitäc zu überstehen.

Damit verbunden ist das höchstrichterliche Attese der verfassungsreclutlichen Unbedenklichkeir der Verwendung unbestimmrer Rechtsbegriffe zur legislativen Konturierung der erforderlichen Umwelcqualität und der zu tolerierenden Risiken (S. I 33 ff.). Ganz ausdrücklich stellt der Senat fest, der Gesetzgeber sei »nicht gehalten, die möglichen Risıkoarten, Risikofakcoren, die Verfahren zu ihrer Ermittung oder feste Toleranzwerte zu bestimmen (S. 139). Gerade dies macht jedoch den Kernbestand aller auf quantifizierte Grenzwerte hin angelegren umweltpolitischen Regelungskonzepte aus ${ }^{18}$. Angesichts der für den Normalbetrieb von Kernkraftwer-

zunchmender materialer - d. h. für ihn vor allem ökonomischer - Ricionalisierungstendenzen (vgl. Wirtschast und Gesellschaft, Tübingen 1972, S , 10 fr.) durch die Ansälze zur "Proxeduralisierung" des Rechts (vgl. dazu R. Wichölter, in: Jahrbuch für Rechessoziologie und Rechtstheorie, Band 8 (1982), S. 8 if.) uad zum wreflexiven Rechea (vgl. dazu G. Teubner, ARSP s8 (is82). S. is ff.) zu verarbeiten versucht.

13 Vgl. dazu Kisker, NJW 77, 1313.

14 BVerIGE 34, 165 (192).

is BVericiE 41, 25 i (260).

16 BVeriCF. 45, $400(417)$.

17 BVerivGE $47,194(197)$

$18 \mathrm{Vgl}$. dazu Koepfel/Weidner, Narmbildung und Implementation. Interessenbericksichugungsmuster in Programmstrukturen von Luftreinhaltepoliniken, in: R. Mayntz (Hrsg.), Implementation politischer 
ken in $\$ 4$ i.V.m. Anhang X, Spalte 2 StrSchV höchstzulässigen Strahlendosen von $30 \mathrm{mrem} / 2$ und der Definition des zu beherrschenden agrößtanzunchmenden Unfalls $_{54}(\mathrm{GAU})$ in den Leitlinien der Reaktorsicherheitskommission's bleibt dic geseczliche Genehmigungsgrundlage, Vorsorge nach dern "Scand von Wissenschaft und Technik « $(\$>$ II Nr. 3 ArG) zu treffen, kaum mehr als eine «aspirative Zielformel ${ }^{20}$ für cine reaktorsicherheitstechnische Programmentwicklung jenseits parlamentarischer Steuerungsinstrumente.

$\mathrm{Da}$ ß damir "Behörden und Gerichte ... das Regelungsdefizit der normativen Ebene ausgleichen w müssen (S. 139), rechtertigt das Bundesverfassungsgericht mit den besonderen Schwierigkeiten, die in der Natur des Regelungsgegenstandes liegen (S. 134). Dem liegt ein Vorverständnis über das Verhälnnis von Recht und Technik zugrunde, das Juristen immer wieder in besinnlichen Wesensschauen über die "Dynamik der Technik" und die "Statik des Rechrs* kultiviert haben". Danach zeichnet sich die Technik durch ihre ständige Innovationsfähigkeit und ihre immer differenzierter werdenden sicherheitstechnischen Anforderungen aus, während dem Recht gerade nicht die Fähigkeit zu decaillierten Regelungen und ständigen Novellierungen, sondern eine auf Dauerhafrigkeit und Allgemeinheit angelegte Regelungsqualität zu eigen sei. Dies mache den legislativen Gebrauch unbestimmter Rechtsbegriffe erforderlich, um den technischen Entwicklungen offene adaptive Scharnierbegriffea zur Seite zu stellen ${ }^{21}$. Die in $\$ 7$ II Nr. 3 AtG enthaltene Genehmigungsformel, kerntechnische Risikovorsorge sei nach dem $\bowtie$ Stand von Wissenschaft und Technik « zu treffen, wird geradezu zum Garancen des posculierten "dynamischen Grundrechisschurzes “ (S. 137) überhöht: „Sie hilft, den Schurzzweck des $\$$ I Nr. 2 A tomG jeweils bestmöglich zu verwirklichen. Die gesetzliche Fixierung cines besrimmen Sicherheitsstandards durch die Aufstellung starrer Regeln würde demgegenüber, wenn sie sich überhaupt berverkstelligen ließe, die technische Weiterentwicklung wie die ihr jeweils angemessene Sicherung der Grundrechte eher hemmen als fördern.\& (S. 1 37 )

Dieser Ansichs liegen zwei Mißverständnisse zugrunde. Zum einen unterschätzt das Bundesverfassungsgericht den hohen immanenten Normierungsbedarf der Großtechnologie, zum anderen überschärzt es die Leitbildfunktion, dic die technikbezogenen unbestimmten Rechubegriffe auf die Entwicklung untergesetzlicher Normwerke und des rechnischen Fortschritts selbst haben können. Hätte das Bundesverfassungsgericht die Struktur der »Normpyramide $\alpha^{23}$, die von der formellen Spitze des A tomgesetzes über Rechtsverordnuagen, Verwaltungsvorschriften, Empfehlungen von Sachverständigengremien, Regeln des durch einen Organisationserlaß des BMI konstituierten Kernrechnischen. Ausschusses bis hin zur privaren rechnischen Normung reicht, kritisch untersucht, wäre die Frage nach möglichen Steuerungsbrüclen im Normentwicklungsprozeß unabweislich geworden.

Programme, Königsteis/Ts. 19\$0, S. 82; R. Wolf, Rechesordnung und Technostruknur. Die Grenzen reguiativer Polirik im Bereich der Kernenergie, in: Jahrbuch für Rechessoziologie und Rechtsetheoric, Band $8(1982)$, S. 240 ff.

$19 V_{g}$ l. dazu RSK-Leidlinien für Druckwasserreaktoren, 2. Ausgabe vom 24. 1. 1979, Koln 1979.

2o Blankenburg/Kmutkrämer, Ein Verwaleungsprogramm als Kaskade von Rechisvorschrifien: Das Arbeisförderungsgesetz, in: Mayncz (FN 18), S. $13^{8}(139)$.

21 Vgl. dazu Breuer, AoR ror (1976), \&6 (f.; Lukcs, NJW 78, 242; Bender, NJW 78, 1945; Backherms, JuS 80, 9; Ossenbüh, DÖV $\$_{2}, 8_{3}$ und meine Krisik in: R. Wolf, Der Stand der Technik. Geschichee, Sinkrurelemente und Funkrion der Verrechulichung technischtr Risiken am Beispiet des Immissiansschurzes, eingereichte Diss. Hannover 1984, S. 270 lf.

22 Vgl. dazu kricisch Ladeur, Leviachan 1979, S. $360 \mathrm{ff}$. und spcxiell /ur das Verfassungsreche Hasc/Ladcur, Verlassungsgerichtsbarkcie und policisches System, Frankfure/Main-Ncw York x980, S. 26, II.

2) Vgl. dazu Becker, DIN-Mite. 78,690. 
In dicsem Zusammenhang ist darauf hinzuweisen, daß der Bedarf an einer Vielzah] von Normwerken zur Regulierung der kerntechnischen Encwicklung unbestreitbar ist. Ihrc Zahl wird heure mit ca. 400 Regelwerken angegeben ${ }^{24}$. Neben einem Duczend Rechtsvorschriften finden sich dort 51 Festlegungen des Länderausschusses für Aromkernenergie. Mit über 200 DIN-Normen und über roo geplanten Regeln des Kerntechnischen Ausschusses und einer Vielzahl von „Emplehlungen « und „Leitlinien * von ministeriellen Beratungsgremien wie Reaktorsicherheirskommission und Strahlenschutzkommission dominieren jedoch dic Vorschriftenwerke substaaticher Organisarionen ${ }^{29}$. Während sich die staalichen Vorschriften auf formell-prozedurale Ablaufstadien der administrativen Bcarbeitung des Genehmigungsverfahrens konzentrieren, definiert das vielfach vernetzte Regelwerk substaatlicher Organisationen die sicherheitstechnisch entscheidenden Risikoschwellen ${ }^{26}$.

Dieses technologiepolitisch zentrale Problem der Auslagerung der Risikobewertung an nichtstaatiche Gremien, in denen durch immer weiter arbeitsteilig vorangetriebene Delegacion und Subdelegation der Normerstellung die einschlägig vinceressierten Kreise u von Reaktorindustric und Energicwirtschaft cine informalc Normungskomperenz über die Dimensionierung von Sicherheitskonzepten und Risikobereichen erhalten ${ }^{17}$, nimmt das Bundesverfassungsgericht nicht zur Kenntnis. Die unbestimmten Rechtsbegriffe haben nach seiner Betrachtungsweise vielmehr auch im technischen Umweltrecht die klassische Funktion, die Feineinstellung der Staatsgewalren zueinander zu justieren. Hier entscheidet sich das Bundesverfassungsgericht, die Beurreilungskompetenz über technische Risiken primär win dic Hand der Exekutive zu geben, deren rechiliche Handlungsformen sie für die eiforderliche Anpassung sehr viel besser ausrüsten als den Gesetzgeber « (S. 139/40). Daß aber dic Genchmigungsbehörden nicht im hinreichenden Maße über hauseigene wissenschaftlich-rechnische Komperenz verfügen, sondern ihrerscirs auf den externen Sachverstand von Wissenschafr, Gutachrerorganisationen, Industrie- und Energiewirtschaft zurückgreifen müssen, veranlaßt das Bundesvcrfassungsgericht nicht zu weiteren staatstheorecischen Überlegungen, obwohl die Konscitution eines nprivate government ${ }^{18}$ der technischen Regulation neben und außerbalb der Staatsorganisation verfassungsrechcliche Bedcnken geradezu auförängt. Statt die Grenzen der durch den unbestimmten Rechrsbegriff "Stand von Wissenschaft und Technik * legitimierten Erosion der polizisch-administraciven Steuerung der Risikodimensionicrung auf der Ebene der Programmenentwicklung abzustecken, deucer das Bundesvcrfassungsgericht auch noch eine mögliche Lockerung der judikativen Kontrolldichte über die Genehmigungsentscheidungen in der Implemencationsphase an, indem es die traditionsreiche unbeschränkte Nachprüfbarkeit unbestimmter Rechtsbegriffe bei der Bewertung rechnischer Normen und Sandards unter Bezug auf dic

$24 \mathrm{Vgl}$. A. Kellemann, Struktur und Zustandekommen sicherbeitsrcicvanter Bestimnungen in technischen Regelwerken für kemtechnische Anlagen, in: Nicklisch/Scholtelius/Wagner, Die Rolle des wissenschats. lich-technischen Sachverstandes bei der Genehmigung chemischer und kemtechnischer Anlagen, Hcidelberg 1982, S. 32 .

is A. A. O.

26 Vgl. daxu Wolf (FN 18)

27 A. a. O. und R. Pieschis, Das Bundesverfassungsgeriche als Verfahrensgeseizgeber - Zur Implemenextion von Verfahrensrecht durch das BVerfG am Beispiel des atomaren Genelimigungsverfahrens, verv. Ms., erscheint in Band 10 des Jahrbuchs fur Rechrssoziologie und Rechestheoric; sowse fur analoge Prozesse im Immissionsschutz A. Hanning, Umweltschuez und iberberiebliche teclmische Nomung, Koln/Berlin/Bonn/München $: 976$.

28 Vgl. dazu allgemein: M. V. Nadel, The Hidden Dimension of Public Policy, Private Govemmenes and the Policy-Making-Process, in: The Joumal of Poliucs 1975, S. 495 ff.; St. Macauly. Privact Government, verv. Ms.. Wisconsin - Madison 19\$3; fur dic Umwelepolirik speziell meine Arbcir *Der Stand der Techinika, a. a. O. (FN 21), S. 134 ff. 
"Vocrde-Entscheidung " des Bundesverwaltungsgerichts ${ }^{29}$ als noch noffen « bezeichnet (S. 136).

Somit bleibt fesczuhalten, daß das Bundesverfassungsgericht offensichtlich an einer restriktiven Kontrolle der prozeßorientierten Dimension der Umweltpolitik zunächst wenig interessiert war. Damit bat es sich auch der Chance begeben, die "Wesentlichkeitstheorie für die Technikkontrolle problcmadäquat zu reformulicren. Wenn es nämlich mitclerweile zum Grunobestand politikwissenschaftlicher

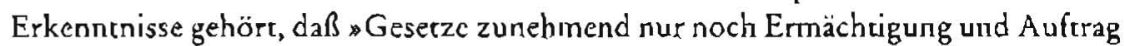
dar(stellen), die Staatstärigkeit von der Verwaltung (und hier auch noch von dem privatcn Interessenten, R. W.) her zu entwickelna', wäre zumindest die parlamenrarische Entscheidung über die Organisation dieses Programmentwicklungsprozesses als "wesentlich « anzuschen, die die Enthaltsamkeit des Gesetzgebers bezüglich der inhaltelichen Qualitäc der politischen Stewerung teilweise kompensieren könnte.

Statt dessen vertritt das Bundesverfassungsgericht die Losung, die Genehmigungsbehörden könnten mit Hilfe der Interpretationshoheit über die unbestimmten Rechtsbegriffe die Risikopolitik bestimmen. Die Basis für die verfassungsgerichcliche Würdigung des Sceverungsgehalts der atomrechtlichen Genehmigungsmaxime vom "Stand von Wissenschaft und Technik « bilder die sogenannce "Dreistufenthese"s'. Danach sollen dic unbestimmecn Rechtsbegriffe allgemein anerkannte Regeln der Technik ( $\$$ 7a WHG), »Stand der Technik ( $\$$; Nr. z BImSchG) und »Stand von Wissenschaft und $\mathrm{T}_{\text {echnik }}$ ( $\$ 7$ II Nr. 3 ArG) drei jewcils unterschiedliche Anspruchsniveaus an dic von den jeweiligen Technologien einzuhalrenden Umweleschurzstandards bezeichnen. In ihnen siehe das Bundesverfassungsgericht auch drei uncerschiedliche Erkenntnisniveaus, indem es für $\$ 7$ a WG lediglich die unter den jeweiligen Praktikern vorherrschenden Auffassungen, für $\$ S $\mathrm{Nr} .2$ BImSchG bereits den Stand fortschritclicher Verfahren und schließlich für $\$ 7$ II Nr. 3 ArG sogar die nichr mehr durch das rechnisch gegenwärcig Machbare zu begrenzende neueste rvissenschaftliche Erkenntnis zum Maßscab nehmen will (S. 135 f.).

Diesem normativen Stufenmodell sceht jedoch entgegen, daß es offensichrlich wenig sinnvoll erscheint, die gleichermaßen unter das BImSchG fallenden genehmigungspflichtigen Enissionen von chemischen Fabrikationsanlagen $(\$ 2$ Nr. I7 4. BImSchV) und der Massentierzucht ( $\$ 2 \mathrm{Nr} .45$ 4. BImSchV) mit den gleichen Filrertechnologien ausstatren zu lassen. Bereits die aufgrund $\S_{4} 8 \mathrm{BImSch} G$ erlassene TA-Luf ${ }^{32}$ modifizierr den abstrakten Maßstab des "Standes der Technik $\alpha$, indem sie erwa Staubemissionen nicht genereil nach dem Grad der abscheidetechnischen Rückhaltbarkeit, sondern nach der Gefährlichkeir der Stoffe differenziert"s". Auch im Vergleich der verschiedenen Normberciche erscheint es völlig unplausibel, warum der Schurz der Gewässer vor Verunreinigungen ( $\$ 7^{\mathrm{a}} \mathrm{WHG}$ ) sich mic einer weniger anspruchsvollen Technik begnügen soll als die Bekämpfung der Luftverschmuızung $(\$$ s Nr. 2 BImSchG). Selbst für den Vergleich mit den Sorgfaltspflichten der Kernrechnik gilr: es wäre unhaltbar, wenn krebsverursachende und erbgutgefährdende Emissionen von Dioxin deshalb weniger streng behandelt werden dürften als die radioakriver Strahlung, weil einmal nur der wStand der Technik «, das andere $\mathrm{Mal}$

\footnotetext{
29 BVerwGL ss, 2 soff.

10 Vgl. F. Schnabel, Policik ohne Politiker, in: Wollmann (Hrsg.), Politik im Dickichs der Burokraric (Leviathan Sonderticfe 3/1979), S. 51 .

31 Vgl. dizu krikisch Nicklisch, B8 83, 261 (263 f.) und Wolf (FN 21), S. 270 ff.

32 GMBl. 1983, S. 94.

3) Vgl. dazu Feidhaus, DVB). 8 y, $165(167)$.
} 
jedoch der ${ }_{2}$ Stand von Wissenschaft und Technik $*$ ( $\$$ II Nr. 3 AiG) gesetzlich vorgeschrieben sei. Wenn das Bundesverfassungsgericht im Anschiuß an dic Rechtsprechung der Verwaltungsgerichte zum atomaren Genehmigungsrecht betont, es müsse »diejenige Vorsorge gegen Schäden getroffen werden, die nach den neuesten wissenschaftlichen Erkenntnissen für erforderlich gehalten wird" (S. I 36), so besrätigt das Bundesverwaltungsgericht für den Immissionsschutz, daß der Schutz vor Gefahren schädlicher Umweltcinwirkungen ( $\$$ Nr. 1 BImSchG) ebenfalls aliein nach dem Stand wissenschaftlicher Erkennenisse über Ausmaß und Eintrittswahrscheinlichkcir der jeweiligen Risikofaktoren zu bemessen seij3.

Allcin vermag auch die im Anschluß an die $"$ Dreifstufenchesen vom Bundesverfassungsgericht im Hinblick auf die "Gefährlichkeir * einer Technologic thematisierte Risikoproblematik nur wenig mehr an rechtsnormativ verdichtbaren Entscheidungsgehalten zu präsentieren. $D a$ aufgrund der jeder Technik notwendig immanenten Risiken ihr Einsatz niemals völlig gefahrlos sein kann's, bleibr immer wieder die Frage, wwie sicher ist sicher genug $\alpha$, zu beanctworten. Kategorische Sicherheitspostulate, die etwa im "Bcrstschutz-Urteila des VG Freiburg mic der Forderung nach der absoluten Beherrschbarkeit einer Gefahr formulicrt wurden ${ }^{16}$, sind damit logisch unhaltbar. Graduelle Konzepre, wie sie zum Beispiel in sog. "Scrahlenminimierungsgebor " (\$28 III StrSchV), die Strahlencxposition der Bevölkerung "sorveic wie möglich“ $z u$ verringern, oder in dem vorn Bundesverfassungsgericht betonten "Grundsacz einer besemöglichen Gefahrenabsvehr und Risikovorsorge" (S. 139) postulierc werden, treffen aber auf quanticative Risikoanalysen und quantifizierte Grenzwertbestimmungen, die die wissenschaftlich-rechnische Risikoforschung erstellt hat. Hier entsteht dann das Problem der direkriven und selektiven Porentiale rechısnormariver Abwägungsformels gegenüber der ingenieurwissenschaftlichen Risikoforschung, den auf dieser Grundlage entwickelten privaten Normwerken und den mit ihnen vemetzten untergeserzlichen Regelungen.

Wenn das aus der "Dreistufenthese enenommene Posculat, im Bereich der Kernenergie nur den "neuesten Stand wissenschaftlicher Erkenntnis" (S. 136) als Kriterium der Genehmigungsfähigkeit anzuerkennen, akzepriert wird, impliziert dies auch eine gesteigerte rechtliche Prüfungspflichs. Sachverständigenaussagen haben für das Bundesverfassungsgericht nicht ohne weiteres die Dignität präsumtiver Richtigkeir, die das Bundesverwaltungsgericht den technischen Regelwerken zuerkannt hatre ${ }^{37}$, vielmehr kommc zumindest die Genehmigungsbehörde »bei sich widersprechenden Sachverständigengutachten in aller Regel nicht umhin, zu wissenschaftlichen Streitragen Stellung zu nehmena (S. 136). Die dafür vom Bundesverfassungsgerichr entwickelten Maßstäbe legen die Vermutung nahe, daß dic Richtersessel in Karlsruhe dem Dreifuß des delphischen Orakels nachgebildet sind. Danach sollen zwar unvermeidliche Restrisiken hinzunehmen sein, während Resischäden hingegen ausgeschlossen werden müssen (S. 137). Der Unterschied dieser sibyllinischen Differenzierung liegt offenbar nur im Grad der Wahrscheinlichkeit. Absehbare Schäden sind nicht zu tolerieren, während nicht mit letzter Sicherheit auszuschließende künfrige Schäden in Kauf zu nehmen sind (a. a. O.). Oder anders gewendet: "Aus verfassungsrechelicher Siche schließt das Gesetz die Genehmigung dann aus, wenn die Errichtung oder der Betrieb der Anlage zu Schäden führt, die sich als Grundrechts-

34 BVerwGE ss, $250(254)$ Voerdex; Feldhaus, DVBl. $81,16 s(170)$ und Horn, UPR $\$ 3,216$.

3s Vgl. dazu P. Marburger. Die Regeln der Teclintk im Recht, Koln/Berlin/Bonn/Múnchen 1979. S. 114 fi.

$36 \mathrm{NJW} 77,1465$ ff.

37 BVerwGE 55,250 . 
verietzungen darstellen ... \$7 Abs. I und 2 Atom G läßr indes Genehmigungen auch

dann zu, wenn es sich nichr völlig ausschließen läßt, daß künítig durch die Errichtung oder den Berrieb der Anlage ein Schaden auftreten wirda (S. 140/4 I).

Den zirkulären Austausch erlaubser Restrisiken und unerlaubter Restschäden versucht der Senat durch den Topos der "praktischen Vernunft zu kaschiercn. Genehmigungen seien nur dann zulässig, wenn es nach dem Stand von Wissenschaft und Technik "praktisch ausgeschlossen erscheint, daß solche Schadensereignisse eintreten wcrden « (S. 143). Darnit fragt sich, ob der Stand "praktischer Vernunft « mehr sein kann als der Standard der Praktiker der ingenieurwissenschaftlichen Risikobewercung. Dies legt zumindest der anschließende Präzisierungsversuch nahe: «Ungewißheiten jenseits dieser Schwelle praksischer Vernunft haben ihre Ursache in den Grenzen des menschlichen Erkennenisvermögens « (a. a. O.). Gerade dies kann aber nicht mehr Gegenseand der naturwissenschaftlichen Risikoforschung sein, die sich nur mit zumindest hypothetisch erkannten Gefahren befassen kann und nach Methoden der Bestimmung der Grenzen der dafür vorzuhaltenden Sicherungen sucht. Damit crweist sich das Bundesverfassungsgericht in seiner Funktion als normkonkretisierender Ersatzgesetzgeber nicht besser als das vielgescholtene Parlament sclbst. Es erliegr dem analogen Zwang zur Produktion von Konsenshülsen und verkünder dilarorische Formelkompromisse.

Der Gehalt des höchstrichterlichen Versuchs, die Entscheidung über die Akzeptabilitär kerntechnischer Risiken durch inhalts- und ergcbnisbezogenc Kriterien bestimmbar zu machen, wird in seinem Scheitern erneur deutlich, wenn man die daran anschlicßenden Operationalisierungsversuche der Verwaltungsrechtsdogmatik betrachret. Hier hat sich ein kaum mehr ubersehbarer Markt konkurrierender Differenzierungsformeln konstituiert, deren Dilemma der begrifflichen Unschärfe nicht nur darin besteht, den Genehmigungsbehörden kaum direktive Entscheidungshilfen bieren zu können, sondern darin kulminiert, daß diese konturenlose Abgrenzungsrhetorik immer wieder konfrontiert ist mit der augenscheinlichen Präzision quantifizierter Grenzwertbescimmungen in untergeserzlichen Regelungen und substaatichen Normwerken. Dic Verbalakrobatik mit Begriffen wie Gefahr, Gefahrenverdacht, Restrisiko und Risikorest, Risiken mic und ohne erkannte Gefahrenqualität, Gefahrenabwehr, Schadensvorsorge, Rest- und Mindestschäden, Risikovorsorge diesseits und jenseits der Schwelle einer praktischen Vorstellbarkeit eincs theorerisch möglichen Schadenseintritts zeigt jedoch nicht nur die wsachliche Hilflosigkeit * der Recheswissenschaf ${ }^{18}$. Diese "juristischen Glasperlenspiele ${ }^{19}$ dokumenrieren auch den Funktionswandel des Rechts in Richrung auf eine inhaitlich denormaxivierte Legitimationsressource, die dazu parallel geführte technische Normensysteme weder in Genesis noch in Geltung kontrollierbar halren kann.

Damit wird auch die Mecamorphose des aufklärerischen Begriffs der sprakcischen Vernunfu", der ja immerbin aus der Kant'schen Erkenneniskritik entstammt und über R. Breuer ${ }^{\circ}$ in die verfassungsgerichtliche Konstruktion der Technikkontrolle Eingang gcfunden hat, zu einer affirmativen Leerformel offenkundig. Ist die Vernunft nach Kant dann prakcische Vernunft, bwenn sie ibren Ideen praktische Realizäi verschaffen kann $\alpha^{41}$, so reduzierr sich ihr Stellenwert in der Judikatur dcs Bundesverfassungsgerichts auf die apriorische Legitimation der programmatisch doch durch

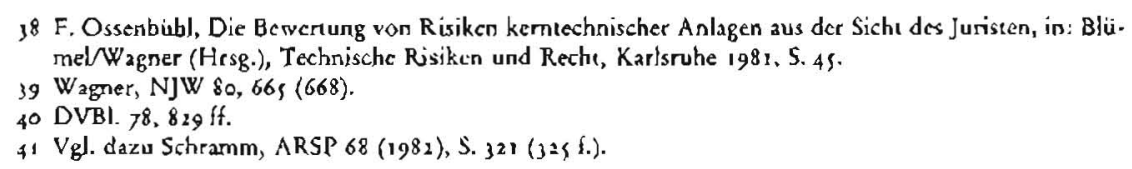


das Recht zu kontrollierenden Entscheidungen im sprivate government " des administrativ-industriellen Komplexes der Kernenergie. Daß die grundrechtlichen Verbürgungen über subjektive Abrvehrrechte des Einzelnen hinaus zugleich objektivrechcliche Wertentscheidungen der Verfassung zur Pflich des Staates darstellen, wsich schützend und fördernd" vor diese Rechtsgüter zu stellen ${ }^{\text {x }}$, hat das Bundesverfassungsgericht zwar gerade auch für die Bedrohung des Lebens und der menschlichen Gesundheit durch die Gefahren der Kernenergie betont (S. ${ }_{4}$ I f.). Daß sich diese Gefahren bisher noch nicht aktualisiert haben, scheint jedoch weniger ein Verdienst des Rechts und der Politik zu sein als vielmehr den immanenten Sicherheitsstraregien der Ingenieurwissenschaften und des glücklichen Zufalls verdanken zu sein.

III. Grundrecble als Verfabrensreche - ein erseer Schritt zu einer

Prozeduralisierung des Umwelcrechis?

Anders als die crgebnisorienticrte „Kalkar-Entscheidung « hat der "Mülheim-Kärlich-Beschluß mit dem Stellenwert der Öffentlichkeitsbereiligung im atomrechrlichen Genehmigungsverfahren ein prozedurales Problem zum Gegenștand (BVerfGE 53, 30 ff.). Hier ging es um die Frage, ob bereits durch einc unterlassene Bekanntmachung und Betciligung Dritter ein Genehmigungsverfahren ungeachtet seines Entscheidungscrgebnisses recheswidrig werden und dic Rechte Dritcer verletzen könne.

Die Rechtsprechung des Bundesverwaltungsgerichts und vieter Obergerichte hatte bis dahin den Standpunkt vertreten, im Regelfall sei eine verfahrensrech:licho Bere:ligung Dritter nichts weiter als ein der Vertvaltung dicnliches Mittel, sich möglichst umfassend über den entscheidungserheblichen Sachverhalt zu unterrichten ${ }^{43}$. Diese etaristische Verfahrenskonzeption war jedoch im Bereich des Umweleschutces ${ }^{\$ 4}$ und speziell auch im Atomrecht"r als probleminadäquat kritisicre worden. Sowohl der VGH Mannheim ${ }^{46}$ als auch das OVG Lüneburgt7 hatten die Neigung zur Abkehr von der bisherigen Rechtsprechungspraxis deutlich erkennen lassen.

Dic verfassungsrecheliche Fundierung des dieser Ansicht zugrande liegenden modernen verfahrensbezogenen Grundrechtsverständnisses hatte insbesondere Häberle mit seinem Konzept des "stacus activus processualis e eingeleitet ${ }^{8}$. Seine am angelsächsischen "due process of law orientierte Grundannahme bcruht auf der Thesc, durch die Berücksichrigung aller relevanten Inceressen geöffnete Vorverfahren vermöchten "mehr Grundrechtswirklichkeit zu schaffen als verwaltungsgerichtliche , Nachverfahren', da im Vorstadium prozessual eingeführt wird, was dem materialen Gehalt des Grundrechts dient«49. Organisation und Verfahren wurden so

\footnotetext{
42 BVeriGE 39, 1 (41) FFristenlösung $\alpha_{;} 4^{6}, 160(164)$-Schleycr $\alpha$.

4) BVCrvGE 39, 23 s: 4 I, 58 (63 6); OVG Rh-Pi GewA 77, 133 (135); Bay VGH DVBI. 79, 673 (677)

$44 \mathrm{Vgl}$. dazu K Redeker, Zur Ausgleichsfunktion von Teilbabereciuen zwischen Freiheit und Bindung, in: Festgabe zum 2 sjährigen Bestehen des Bundesverwaltungsgerichts, Mùnchan 1978, S. fi ( (20); Breuer NJW $78,1598(1564)$.

4) $V_{g}$. die Bcitrage von Blümel und Kimminich, in: Lukes (Hrsg.), Finfies Deutsches Atomreches-Symposium, Berlin/Koln/Bonn/München 1976, S. 227 fl. und $266 \mathrm{ff}$.

46 DVBI. $76,33^{8}(540) *$ Whyls

47 DVBI. 77, 347 (348) *Dok Chemical\%.

$4^{8}$ Vgl. dazu P. Habcrlc. VVDStRL 30 (1972), S. 45 (86 if.); Hesse, EuGRZ 78, 427 und nun crweiternd

H. Gocrlich, Gundrechee als Verfahrensgarancien, Baden-Baden $198_{3}$

49 Haberle, a. 2. O., S. 88 .
} 
zunehmend von der neuen Grundrechtsinterpretation entecks und aufgerver-

tet ${ }^{50}$.

Da das Bundesverfassungsgericht in vorsichtiger Rezeption dieser Ansätze bereits in früheren Entscheidungen betont hatte, ${ }^{2}$ daß Grundrechisschucz weitgehend auch durch die Gestaltung von Verfahren zu bewirken ist und daß die Grundrechte demgemäß niche nur das gesamte materielle, sondern auch das Verfahrensreche beeinflussen " ", lag cs nahe, nach der im "Kalkar-Beschluß " beconten objektiv-rechtlichen Schurzp flicht des Staates in materieller Hinsicht, die Verletzung subjekriver Rechte auf Verfahrensteilhabe dann in Erwägung zu ziehen, w wenn die Genelımigungsbehörde solche Verfahrensvorsch riften außer acht läßt, die der Staat in Erfüllung seiner Pflichr zum Schutz der in Art. 2 Abs. 2 GG genannten Rechtsgüter erlassen hat (BVerfGE \{3, 30 (65/66)). Dies bejahi der Senat zunächse ganz pauschal für dic Öffentlichkeitsbeteiligung am atomrechtlichen Genchmigungsverfahren (S. 66).

Daß der Verfassungsbeschwerde dennoch im Ergebnis kein Erfolg beschieden war, liegt an der anschließenden Bewertung dieser Rechte für die im Rahmen der nach $\$ 80 \mathrm{~V}$ VwGO gebotenen Interessenabwägung im angegriffenen verwaltungsgerichtlichen Eilveriahren. Diese schwer nachvollzichbare Relacivicrung der eben prokJamierten Verfahrensrechte hat auch die Richter Simon und Heußner zu ihrem vielbeachteten Minderheitsvotum veranlaß\}t (BVerfGE 53,69 ff.). Darin wird die Bedeutung von Verfahrensrechten dahingehend prononciert, dic staatlichen Organc hätten wnicht nur Pflichr, die materiellen Grundrechte zu beachten, sie müßcen ihnen auch durch eine entsprechende Verfahrensgestaltung Wirksamkeit verschaffen (S. 72). Intention des Minderheitsvotums scheint zu sein, die durch ausfüllungsbedürfige Normbegriffe im materiellen Genchmigungsrecht entstehenden Unsicherhciten für die gemäß Art. 2 II GG zu schützenden Rechtsgüter durch verstärkte prozedurale Kontrollen zu kompensieren. Der Gebrauch unbestimmer Rechtsbegriffe erschiene weher tragbar, wenn durch ein formalisiertes, gerichtlich kontrollierbares Verfahren dafür vorgesorgt werde, daß die wesentlichen Entscheidungsfaktoren geprüft und die mit der Norm angestrebten Ziele wirklich erreicht würden* (S. 75) $)^{\prime 2}$. Gerade wenn aufgrund des Regelungsuefizits der gesetzlichen Programme die Definition von Sicherhcits- und Risikodimensionen auf die administrative Ebene verlagert werde, könne es nverfassungsrechtlich nicht gleichgültig scin, wie gerade auch das behördliche Genehmigungsverfahren strukturiert ist und wer als Beteiligter auf die Entscheidung Einfluß nehmen kann * (S. 76). Somit vertrauen dic Richrer auf umfassende Informacionsgewinnung durch offene Kommunikation als Rationalitätsgarantien des Encscheidungsprozesses. „Daß dieser Kommunikationsprozeß nur bei einem allscits fairen und vernünfrigen Verhalten funkrionieren kann, liegt auf der Hand * (S. 77).

Offenkundig ist jedoch auch, daß dieses normative Verfahrensmodell gerade im Umweltschuzz durch informale Aushandlungsprozesse allerorts unterlaufen wird's und daß die so stark gewichcete Öffentlichkeitsbeteiligung in Genehmigungsverfahren gerade nicht zum Garanten der Rationalität, sondern zu den Mustern gestörter Kommunikarion zu zählen ists`. Zumindest bei polygonalen und multidimensiona-

\{0 Vgl. zusammenfassend Hesse, EuGRZ ;8, 427.

\{1 So BVeriGE \{3, 30 (6s); schon früher zu Arr. i4 GG: BVerfGE \}7, $132(141$ ff.); 46,32$\}(334) ; 49,220$ (225), zu Ari. 12 GG: BVerfGE 39, $276(296) ; 44,105(119) ; 45,422$ (430 [f.); 52. 381 (389); zu Art. 2 GG: BVeriGE $\{1,324 ;\{2,2\} 4$ "R̈umengsschurz .

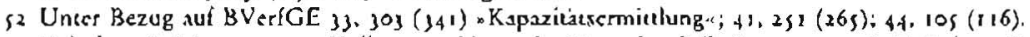

s3 Vgl, dazu R. Mayntz, u. a., Vollzugsprobleme der Umweltpolitik, Stungart 1978; E. Bohne. Der informale Rechesstaxt, Berlin 1981 .

$\$ 4$ Vgl. Batclle-Institut, Der Eröncrungseemin, Frank(urt 19so 
len Verwaltungsentscheidungen mit makro-administrativen Auswirkungen erweist sich das von Ule/Laubingerss und Häberles6 besonders emphatisch vertretcne Harmonicmodell des partizipatorischen Verfahrens als eine Hypothese, deren Fragwürdigkeit bercits ein Blick aư soziologische Verfahrenstheorien bekuäfrigt hätte ${ }^{37}$. $\mathrm{Zu}$ Recht resümier Hoffmann-Riem über den Zusammenhang von Verrechtlichung und informalen Umgehungsstrategien: sInformelle Ausweichhandlungen dürfien um so wahrscheinlicher sein, je größer der Aufwand für die Einhaicung rechtlicher Formalisierungen wäre, je übcrflüssiger er von den Beteiligten empfundev würde, und je mehr dic Formalisicrungen einem realen oder vermuteten Flexibilitätsbedarf widersprächen ${ }^{58}$. Ihre Folgen sind um so einschneidender, je deuclicher die Asymmetrie zwischen den Beziehungen der Genehmigungsbehörden und der Antragsteller auf der einen Seite und den einwendeführenden Dritzbetroffenen auf der anderen Seite auffällt. Zugleich mit der Betonung der Verfahrensrechte siehc sich damic die Rechtswissenschaft vor das Folgeproblem gestelle, Kriterien für cine Mißbrauchsgrenze informaler Kooperationsbeziehungen und für andere Umgehungsversuche formaler Bindungen zu entwickeln"s. Solange das soziale Machtdifferential unter den Akıuren der Umweltpolitik nicht an den Grundstrukturen der sciektiven Interessenberiicksichrigungsmuster von Programmentrvicklung und Implemencation themacisiert wird, erscheint die Bctonung von Verfahrensrechten, die am Ende des politisch-adminiscrativen Entscheidungsprozesses crst wirksam werden können, als Versuch, das Pferd vom Schwanz her aufzuzäumen. Die zahlrcichen informalen Umgehungsstrategien widerlegen jedoch keincswegs die Erkenntnis von der Grundrechssrelevanz von Organisation und Verfahren, sondern bestätigen sie gerade. Aus den sich ihnen entgegen stellenden Widerständen kann zumindest entnommen werden, daß eine prozeßorientierce Umwelsschutzkonzeption verfesrigze Macht- und Einflußkonstellationen durch die eingeleitere Politisierung des Entscheidungsvorgangs zu irricieren vermag.

Mit der These, "daß sich wahrscheinlich nur über das Vorfahrensrecht verhindern (lasse), daß der Bereich zrvischen Recht und Technik zum juriscischen Niemandsland wird" (S. 76), weist das Minderheirsvotum schließlich auch über den zu eng gefaßiten Rahmen der prozeduralen Grundrechtsgarantien im atomrechtichen Genehmigungsverfahren hinaus. Gefragt wirỏ nach der "Zusammensetzung und Vedfahrensweise solcher Kommissionen, die allgemeine Sicherheitsrichtlinien oder Immissionsgrenzwerte erarbeiten und damit weitreichende Entscheidungen präjudizieren « (S. 78). Damit wird zum ersten Mal die polirische Qualicät der atomrechrlichen Programmenrwicklung aus prozeduralen Grundrechtserwägungen heraus in Frage gestellt ${ }^{60}$. Berücksichtigt man jedoch die rcstriktive Rezeption des $*$ MülheimKärlich-Beschlusses « in der Rechtsprechung des Bundesverwaltungsgerichts ${ }^{61}$ und die reserviexte Verfolgung des Verfahrenskonzepes in der anschließenden Rechtsprechung zum aromaren Genehmigungsrecht durch das Bundesverfassungsgericht selbst $^{6_{2}}$, so schcint eine Weiterführung der im Minderheitsvotum enthaltenen Kontrollansärze angesichus der Pendelschläge der Rechtsprechung zwischen vorwärts-

\footnotetext{
is Ule/Laubinger, s2. DJT, B 216 .

s6 In: Festschriff lür Boorberg-Verlag: Sruxtgan-München-Hannover 1977, S. ${ }_{4} 7 \mathrm{H}$.

37 N. Lulumann, Legitimation durch Verfahren, Berilin-Neuwied 1967.

58 VVDStRL $10(1982)$, S. $190(226)$.

59 Vgl dazu BVerwGE 45, 309 . Floatglasu und Hoffmas-Ricm, a. a. O., S. $217 f f$

to V Vl. dizu auch Gocrlich, a. a. O., S. 280 u. 283 ; Woll (FN 21), S. 444 II.

6. BVcrowGE 41, $88(63) ; 60,296(275) ; 62,243(246)$.

62 BVeriGE 6r, 81 ( $110 \mathrm{u} .113)$. Vgl. zur Kricik der Tendenzen \%ur prozessualen Abschoutung der Vcrowal. tungsgerichte Geulen KJ 80, 170 und Bosselmann KJ 80, 389: $81,402$.
} 
weisenden Ansätzen und affirmativer Reorientierung an den geronnenen bürokratischen Handlungsmustern über die Rechtsfortbildung allein nur schwerlich realisier$\mathrm{bar}^{6}$.

IV. Grundrechte als Anspruch auf Gesetzgebung - eine enweiterte Perspektive der Prozeduralisierkng des Umwelirechts?

Der "Fluglärm-Beschluß (BVerfGE $\leqslant 6,54$ ff.) kann als Weiterführung der in der "Mülheim-Kärlich-Entscheidung * aufgeworfenen prozeßoricntierten Rechrmäßigkeirskontrolle verstanden rverden. Statt der Öffentlichkeitsbereiligung bei der administrativen Implementation von Umweltgesetzen steht nunmehr die parlamentarischc Programmentwicklung selbst im Brcnnpunkt verfassungsrechtlicher Erörterungen.

Was im »Kalkar-Beschluß « noch vom Zweiten Senar für den „Schnellen Brüter a aus der "Natur der Sache für ohne rechtliche Altemativen erklärt worden war, gewinnt nun für den Ersten Senat an rechtsnormativ zu steucrndem Problemgehalt: die Regelungsabstinenz des Geserzgebers, seine nur vagen inhaltlichen Programmaussagen und deren zweifelhafte Aktualitä.

Wie an fast allen Problembereichen der Umwelrpolitik zu beabachten, stcht auch die Regularion der Lärmbelastung durch den Flugverkehr in einem deutlichen Mißverhàltnis zwischen der Extensität der formellen Verrechtlichung und der unzureichenden inhaltlichen Regelungsintensitär dieser Normen. Dies konnre jedoch nicht mehr unmittelbar Gegenstand der materiell-rechtlichen Prüfung des Bundesverfassungsgerichts sein, da die Verlassungsbeschwerde gegen die Zulassungsregeln von Verkehrsflugzeugen im Luftverkehrsgesetz von 1959 und gegen die Immissionswertkonzeption des Fluglärmgesetzes von 1971 wegen Versäumung der durch $\$ 93$ II BVerfGG limitierten Frist verwirkt war. Das Bundesverfassungsgericht erörtert demgemäß auch nur, ob der Gesetzgeber durch Untärigkeit angesichts allgemein zunehmender Flugbewegungen, des Überganges zu lautstarken Düsenmaschinen und der zunehmenden Besiedelungsdichte nicht eine verfassungsrechtliche Pfliche zur Nachbesserung einer ursprünglich als verfassungskonform zu betrachrenden Regelung haben könnte (BVerfGE s6, 70 ff.). Dogmatischer Bezugspunkt ist dabei wie bereits für die eben diskutierten Entscheidungen dic aus Art. 2 II GG hergeleitete objektiv-rechtliche Schutzpflicht des States, Leben und Gesundheit vor rccheswidrigen Eingriffen zu bewahren (S. 73).

Daß dieser Rechtsgedanke zum ersten Mal in der Encscheidung zur "Fristenlösung * (BVeriGE 39, I (4I)) formuliert wurde, mag überraschen. Der rechtsdogmatische Bezugsrahmen dieser viel gescholtenen Judikatur erweist sich nunmehr als potenticller Hebel, die Versäumnisse der Umweltpolitik justizförmig zu thematisieren. Kann man die Inscrumentalisierbarkeit des verobjektivierten Schutzbereichs der Art. I und 2 GG für die Belange des Umweltschuizes noch mir der Hegel'schen "List der Geschichrea ironisieren, so verstärkt sich damit jedoch auch die Tendenz 7ur Deparlamentaricierung des Verfassungsreches.

Trotz des grundsätzlich einer richterlichen Nachprüfung entzogenen politischen Ermessens des Gesetzgebers bei der Entscheidung über den Erlaß eines Gesetzes ${ }^{6_{4}}$ gehörr die Bejahung einer Nachbesserungsplicht für einmal erlassene Geserze mitr-

6) So auch Pitschas (FN 27), S. 26

64 BV criGE 1. 97 (100); 11, 25s (261) 
lerweile zum Grundbestand der verfassungsgerichtichen Kontrolle der politischen Programmentwicklung ${ }^{6}$. Der vielfach zu beobachenden geschichrslosen Dethematisierung dieser demokratierheorerisch schwerwiegenden Entwicklung sei immerhin die kritische Anamnese gegenüber gestellt, daß Laufer noch 1968 eine solche Unterlassungskontrolle des Gesetzgebers in den Bereich utopischer Phantasmagorie verwiesen hatte ${ }^{66}$. Daß sich das Bundesverfassungsgericht nun mehr und mehr als politische Initiativkörperschaft gerien ${ }^{67}$, erscheint aber heutzutage nur noch dann dubios, wenn man einem illusionären Parlamentarismusbegriff anhängr, der der Verfassungsgerichtsbarkeit keinen maßgeblichen polirischen Gestaltungsspielraum zuerkennen will. Im übrigen erscheint cine durch das Verfassungsgeriche ausgesprochene Verpflichrung des Gesetzgebers zur bisher versäumten Gesetzgebung demokratietheoretisch weniger brisant als eide restriktive inhaltiche Zensur der von ihm getroffenen Entscheidungen, da damic lediglich ein parlamentarisch dethematisiertes Problem an die Legislative zurück adressier wird.

Bereits in der «Kalkar-Entscheidung " waren die Maximen der parlamentarischen Nachbesserungspfliche grundsärzlich auch für den Umwcluschutz als anwendbar erklär worden. Eine Pflicht zum wNachfassen" sei insbesondere bei wnoch nicht absehbaren Entwicklungen " und "veränderten Umständen a zu erwägen (BVerfGE 49, 130). Entsprechend konstarierte auch der »Mülheim-Kärlich-Beschluß cine staatliche Mitverantwortung für die Risiken der modernen Technologien, w wenn der Staat durch die Schaffung von Genehmigungsvoraussetzungen und die Ertcilung von Genehmigungen « räcig geworden sei (BVerfGE 33, 58). Dem tric nunmehr das Gerichı auch für das Problemfeld des Fluglärms bei, indem es in ihm grundsätzlich cin den Schutzbereich des Art. 2 II GG tangierendes Gesundheicsrisiko anerkennt (BVerfGE , 6, 77) und die nach wissenschaftlichen Erkennenissen gebotene Lärmbekämpfung zu einer grundrechtsrelevanten Pflicht erklärt, die "nicht ausschließlich davon abhängen (dürfe), welche Maßnahmen gegenwärtig technisch machbar« seien (5. 80).

Obwohl der Senat weiter konstatiert, daß zul dem Gebier der Fluglärmbekämplung weder befriedigende Verhäknisse erreicht noch alle denkbaren Schutzmaßnahmen verwirklicht seien, kornmt er gleichwohl zu dem Ergebnis, der Gesetzgeber habe die ihm obliegenden Schurzpilichten durch das Unterlassen von Nachbesserungen am Gesetz noch nicht verleczr (a. a. O.). Es fehle nämlich an einer evidencen Pflichrverletzung, die das grundsätzlich zu beachtende politische Ermessen des Gesetzgebers so reduziert haben könnte, daß sich der Entscheidungsspielraum über das "Ob $\mathrm{O}$ und * Wier umweltpolitischer Interventionen gleichsam alternativlos verdichtet hätte ${ }^{68}$. Ein Verfassungsverstoß kann danach erst dann festgestellt werden, swenn evident ist, daß eine ursprünglich rechtmäßige Regelung wegen zwischenzeitlicher Änderung der Verhältnisse verfassungsrechrlich uncragbar geworden ist, und wenn der Geserzgeber gleichwohl weiterhin untätig geblieben ist oder offensichelich fehlsame Nachbesserungsmaßnahmen getroffen hat" (S. $8 \mathrm{r}$ ).

So plausibel die vom Bundesverfassungsgericht entwickelten allgemeinen Kriterien für eine judikative Kontrolle der parlamentarischen Programmentwicklungs- und Programmrevisionspflicht zunächst auch ausfallen mögen, so wenig kann die darauf folgende Subsumtion überzeugen. Hier härte der Scnat im einzelnen klären müssen,

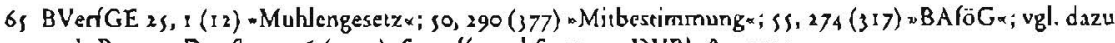
auch Breuer, Der Staat 16 (1977). S. 21 If. und Sietinex, DVBI. 83, 1123.

$66 \mathrm{H}$. Lauler, Bundesveriassungsgerich, und politischer Prozeß, Tübingen 1968, S. 371.

$67 \mathrm{Vgl}$. daxu Massing (FN s), S. 42 .

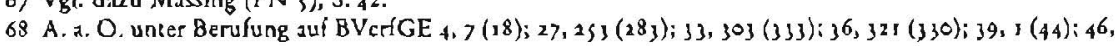
$160(164)$. 
ob dic in den einschlägigen Gesetzen enthaltenen Regelungskonzepte, Eingriffsbe-

fugnisse und Exmächtigungen zur untergesetzlichen Programmentwicklung als legislative Steuerungsdimensionen ausreichen und somit eher ein administratives Vollzugsdefizit anzunehmen ist oder ob es hinreichende Gründe gibt, die Konzeptualisierung des Fluglärmschutzes durch den Geseczgeber selbst zu kritisieren. Bedenken härten sich hier vor allem wegen mangelnder gesetzlicher Vorgaben für einen aktiven Lärmschutz aufdrängen müssen. Statt dessen begnügte sich das Bundesverfassungsgericht mit der Exkulparion der parlamentarischen Regelungsabsrinenz durch die Zusammenstellung von Aktivitäten, die von internationalen Gremien, der Bundesregierung und vom Bundestag seit 1971 eingeleiter worden waren (S. 82 ff.). Geht es um das Untäcigbleiben des Gesetzgebers, so erscheine der Verweis auf die umfangreichc Förderung der Umweltforschung, die Berufung von Konmissionen, die Erstellung von Bestandsaufnahmen zur Umweltbelastung, die Veranstaltung öffentlicher Hearings und parlamentarischer Anfragen allein als keinc ausreichende Begründung für die dilatorische Behandlung des Problems als Gegenstand der parlamentarischen Gescizgebung. Statt dessen scheint sich darin weniger der Regelungswille als vielmehr die aktivistische Version der Politik der »non-decisions “ zu dokumentieren.

Eine Parallele zum Regelungsdefizir im Fluglärm bieret das Problem des Verkehrslärms, auf den das Judikat des Bundesverlassungsgerichts übertragbar ist. Hier sah zunächst das Schutzsystem der $\$ \$_{4} 1$ ff. BImSch G eine weitgehend auf die administrative Programmentwicklung delegierte Ermächtigung vor, durch den Erlaß von Immissionsgrenzwerten im Verordnungswege die erforderlichen quantitativen Festlegungen zu treffen. Entsprechende Arbeiten der Ministerialbürokratie wurden jedoch abgebrochen, um cin umfassenderes Verkchrslärmschutzgesetz zu ermöglichen. Nach seinem parlamentarischen Scheitern blieben die $\$ 4$ If. BImSchG nicht weiter operationalisierte Regelungsrudimence $e^{69}$. Nachdem nun das Bundesverwaltungsgericht die Unvollziehbarkeit der Verkchrslärnsschutzkonzepte festgestellt hat $t^{\circ}$, liegen verfassungsrechtliche Bedenken nahe, ob der Staat nicht gegen die vom Bundesverfassungsgericht reklamierte Schuczpflicht verstoßen hat. Stellt man auf die Regelungsbedürítigkeit und die Regelungsabscinenz ab, liegt eine Verleczung des Art. 2 Il GG auf der Hand, läßt man die reine politische Aktivität im vorlegislativen Raum genügen, käme man zu cincm dem »Fluglärmbeschluß« entsprechenden Ergebnis.

Die mangelnde Stringenz der verfassungsgerichelichen Programmennwicklungskontrolle hat für die Bezichungen zwischen Parlament und Verfassungsgerichtsbarkeit ambivalente Folgen. Zum einen dokumentiert das Bundesverfassungsgericht in der grundsätzlichen Bejahung von Nachbesserungspllichten des Parlaments, daß es gewillt ist, den Gesetzgeber unter verstärkte Kontrolle zu stellen, zum anderen zeigt seinc bisherige Enuscheidungspraxis, daß der akcuellen Umweltpolicik von dem demonstrativ über das Parlamenr gehängten Damoklesschwert derzeit noch wenig zu drohen scheinc. Die Entscheidung des Bundesverfassungsgerichts zur Verfassungsbeschwerde gegen das staatliche Untäcigbleiben gegenüber dem wsauren Regen « belegt diese These"'. Somit entwickelt das Gericht auch gegenüber der legislativen Programmentwicklung im Umweltschurz weniger die Attitüde des auroritä-

69 Vgi. daz.u E Schmidt-ABmann, Schutz gegen Verkehrslärm, in: Salywedd (Hrsg.), Grundzüge des Umwelirechis, Berlin 1982, S. 313 (318).

70 BVenvG DOV 81, $75^{8}$ (759).

7 BVerfG UPR 83, 372. Kaum ein Bereich des Umweltrechts schcint in Zukunf vor ähnlichen Verfassungsbeschwerden sicher zu scin. Eine niue Beschwerde in Sachen Dioxin ist bercies anhängig (vgl. Der Spiegel $25 / 19 \&_{4}$ ) 
ren Zensors als der milden Autorität cines souveränen Hüters des verbände-pluralistischen Konsenses ${ }^{22}$.

Jedoch ist anzunehmen, daß mit der Verschärfung der ökologischen Krisensymprome und dem verstärkten Auftreten organisierter Interessen zum Schurze der Umwelt die in der "Fluglärmentscheidung" entwicketren Ansäıze nicht folgenlos bleiben können. Bedeutungsvoll erscheinen sie aber weniger in bezug auf eine Programmentwicklungskontrolle des Bundestages als vielmehr der Exekutive und hier besonders der einzelnen Länder. Betrachtet man die vielfältigen und zum Tcil jahrzehritelang ungenutzten Ermächsigungen zur umweltpolitischen Programmentwicklung durch die Regienungen der einzelnen Bundesländer", so ist diese Regclungsabstinenz um so prekärer, als aus den allgemeinen Zielen der jeweiligen Gesetze und ihren zum Teil bindenden Ermächtigungsnormen ein im Vergleich zu Art. 2 II GG wesentlich bestimmerer Schutzauftrag zu entnehmen ist. Allein die Restriktionen des auf den Individualrechtsschurz. reduzierren öffentlich-rechtlichen Rechtsschurzsystems können hier noch der Mobilisierung einer umfassenden judikativen Programmentwicklungskontrolle entgegengehalten werden.

\section{Umweltschutz als Inhalts- und Schrankenbestimmung des Privateigentums - neu enedeckıe Grenzen für die Grundrechtsausübung?}

Der "Naßauskiesungs-Beschlußa (BVerfGE $s, 300$ ff.) enthält in seiner umweltrechtlichen Komponente einen Abgrenzungsversuch zwischen verfassungsrechtlichen Bestands- und Entschädigungsgarantien des Privateigentums und den Gestaltungsspielräumen der Umweltpolirik. Insoweit unterscheider er sich von den technikzentrierten Entscheidungen, die eben erörrert wurden.

$\$$ ra III des WHG normiert einc für den Umweleschuez richrungsweisende Schrankenbestimmung des Privareigentums: „Das Grundeigentum berechtige niche I. zu einer Gewässerbenutzung, die nach diesem Gesetz oder nach den Landeswassergesetzen einer Erlaubnis oder Bewilligung bedarf ...* Daruncer fällt nicht nur die Einleitung von Abwässern, sondern auch die Kiesgewinnung, soweic dadurch das Grundwasser berühre ist. Der damir intendierte Gewässerschuzz wurde durch die restriktive Rechrsprechung der Venwaltungsgerichtsbarkeit zur behördlichen Gestattung solcher Eingriffe in die Natur unterstützt ${ }^{74}$, durch die entschädigungsrechtijche Judikarur des Bundesgericheshofes"s im Falle der Versagung der wasserrechtlichen Genehmigungen jedoch auf der fiskalischen Ebene fakrisch weirgehend konterkariert ${ }^{6}$.

Demgegenüber stelıt das Bundesverfassungsgerichr im nNaßauskiesungs-Beschluß $\beta_{*}$ ausdrücklich fest, das WHG unterstelle „das Grundwasser einer vom Grundeigen-

72 Vgl. dazu Hase/Ladeur (FN 12), S. 314 If.

73 Vgl. nur $\$ \$ 36,16 \mathrm{~b}, 27$ WHG; $\$ 15$ III AbFG; $\$ \$_{44}, 46,49$ I, 49 II, 49 III BImSchG.

74 Vgl. BVerwGE $\$ \$, 220$

75 BGHZ 60, 126.

76 Dic justizimmanente Bedeutung dieses Beschlusses licgt daher in der emcuten Karstellung der Kompa tenzbereiche der ordentlichen und der Verwalnungsgerichesbarkeit bei Rechusstreitigkeiten um den Eigentumsbegriff des Arr. 14 GG. Daß dicser Encscheidung der juscizpolicisch àvßerst delikate Vorgang eines vom BVerfG bejm dissentienen BGH nachdrucklich angemahnten Vorlagebeschlusses zugrunde liegt (vgl. dazu das obiter diccum in BVerfGE 45,63 (75 f. u. 80 (.) und den VorlagebeschluB (BGH NJW $78,2290)$ ), um dic entgegenlaufende Rechisprechung des BGH in dic verfassungsrechulichen Schranken zu weisen (vgl. erwa BGHZ 60, 126 (136 (.))), sei hier nur angemerkt (vgl. da774 Bryde. Der Kampi um dic Definition von Ant. 14 GG, verv. Ms., erscheint in Band 10 des Jahrbuchs für Rechessoziologiv und Rechestheorie; zur kontroversen Rezeption von BVerfGE 88, j00: Rittsticg, NJW' 82, 721; Kimminich, NuR 83 , s; Leisner, DVBI. ${ }_{3}, 6$,.) 
tum losgelösten öffentlich-rechtlichen Benutzungsordnung " (S. 328). Dem entspricht auch der Regelungsansatz des $\$ 6$ WHG, nach dem den Nutzungsinteressenten grundsätzlich kein Rechtsanspruch auf Genchmigung ihrer Vorhaben zusteht (a. a. O.). Somit basiert das Eigentumskonzept des Wasserrechts darauf, daß $\triangle \mathrm{dem}$ Grundsrückseigentümer prinzipiell nichs das Rechr gewährc (wird), auf das im Untergrund vorhandene Wasscr einzuwirken. So wie seine Befugnisse an den Grundsrücksgrenzen enden, endet seine Rechtsstellung in die Tiefe prinzipiell dort, wo er mic dcm Grundwasscr in Bcrührung komme (S. 329).

Für das Bundesverfassungsgericht gehören diese wasserhaushaltsrechtlichen Rescrikrionen zu der gemäß Ar. ${ }_{4}$ I 2 GG legislativ zu konkretisierenden Definition des Inhalts und der Schranken des Eigentums, die deutlich von der Legal- und Administrativenteignung des Art. 14 III 2 GG abzugrenzen sei (S. 330 f.). Mithin liegt im zivilrechtlichen Eigentumsbegriff keine abschließende Regelung von Inhalt und Schranken der verfassungsrechtlichen Eigentumsverbürgungen des Arr. i 4 GG. Dies bedeutet auch die Absage an die aus den justizpolitischen Kampfzeiten der Weimarer Republik tradierte Auffassung", eine Enteignung liege schon dann vor, "wenn das Recht des Eigentümers, mit seiner Sache gemäß $\$ 903$ BGB nach Belieben zu verfahren, zugunsten Dritter beeinträchtigt werde $^{7^{8}}$. Dazu stellt das Bundesverfassungsgericht lapidar fest: Diese am Vorrang der bürgerlich-rechtlichen Eigencumsordnung gegenüber öffentlich-rechtlichen Vorschriften orientierte Rechtsansicht entspricht nicht dem Grundgesetz. Der Begriff des von der Verfassung gewährleisteten Eigentums muß aus der Verfassung selbst gewonnen werden $\alpha$ (S. 335 ). Damit schwindet auch die Begründbarkeit des Eigentumsschutzes gegenüber der Umweltpolitik aus vorgeblich überpositiven und vorstaatlichen Wertordnungen. - Der Gesetzgeber ist bei der Schaffung einer dem Grundgesecz gemäßen Güterordnung nicht an einen aus der, Nacur der Sacher sich ergebenden Eigentumsbegriff gebunden « (S. 339). Gesetzgebungstechnisch bedeutet dies, daß bei knappen Umweltressourcen das präventive Verbot mit Erlaubnisvorbehalt, das einen Rechtsanspruch auf Genehmigung einräumt, wenn keine Versagungsgründe greifen, nicht mehr als das einzig adäquate ordnungsrechtliche Komplementärinstitur zum Privateigentum betrachern werden kann.

Die möglichen Folgen dieser Entscheidung sind noch nicht absehbar. Was das Bundesverfassungsgericht in bczug auí den Grundwasserschutz entwickelt hat, wird für den Schurz fließender Gewässer prinzipiell kaum zu ncgieren sein. Fernwirkungen auf andere Rechtsgebiete, insbesondere zum Immissionsschutz, sind, zumal das Bundesverfassungsgerichr explizit auf die Vergleichbarkeit des Wasserhaushalts und der Luftreinhaltung verweist (S. 339), gleichfałs kaum von der Hand zu weisen ${ }^{79}$. Davon könnten grundsätzlich nicht nur neu zu errichtende Anlagen betroffen werden, sondern auch bereits konzessionierte Altanlagen. Schon für alte wasserrechtliche Nutzungen hat das Bundesverfassungsgeriche betont, der Gesetzgeber sei bei der Neuordnung eines Rechtsgebieres nicht gehalten, »die alten Rechespositionen zu konservieren oder gegen Entschädigung zu entziehen« (S. 351 ). Vielmehr könne er durch weine angemessenc und zumurbare Überleitungsregelung individuelle Rechtspositioncn umgestalten, wenn Gründe des Gemeinwohls vorliegen, die den Vorrang vor dem... Vertrauen auf den Fortbestand eines wohlerworbenen Rechts verdienen $4(S .351)^{80}$.

\footnotetext{
77 Vgl. dazu O. Kirchheimer, Die Grenzen der Enceignung (1930), in: ders., Funkzionen des Staates und der Veriassung. 10 Analysen, FrankfuruMain 1972, S. 223 fl.

78 RGZ 116,268 (272).

$79 \mathrm{~V}_{\mathrm{g}}$. dazu den schr inseruksiven Aufsalx von Sendler, UPR 83, 33 (40 f.),

80 Unter Hinneis auf BVerfGE 31, 275 (285); 36, 281 (293): 43, 242 (288).
} 

prozeßorientiertes Umweltrecht?

Vergleicht man die vier umweltrechtlichen Leitentscheidungen des Bundesverfassungsgerichts, ergibr sich ein Schwerpunkt in den rechnikbezogenen Problematisie-

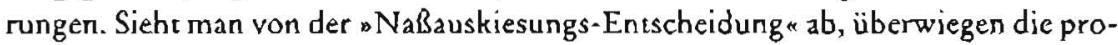
zeßorientierten Thematisierungen die ergebnisorientierten. Darüber hinaus hat auch die ergebnisorientiertc Fragestellung des „Kalkar-Bcschlusses « kaum die materiellen Rechtmäßigkeirskriterien entwickeln können, auf die eine inhaltliche Kontrolle rechnischer Risiken angewiesen ist. Dagegen enthalten der *Mülheim-KärlichBeschluß und die "Fluglärm-Entscheidung* eirige wichtige und ausbaufähige Überlegungen zur Dimensionierung des verwaltungs- und verfassungsgerichtlichen Prüfungsrahmens, da sie Programmentwicklung und Implementation von Umweltstandards als politisch-adminiscrativen Prozeß begreifen, für den ergebnisbezogene Rechtmäßigkeitserwägungen nur in seltenen Fällen eindeutige Maßstäbe scızen können. Allerdings bleiben auch die prozedural orientierten Ansätze letztlich solange noch Kontrollrhetorik, als sie nur abstrakte Kriterien entwickeln, ohne sie im konkrecen Fall prakcisch werden zu lassen.

Nimmt man die eigentumsrechdiche Komponente der Umweltpolitik, die der *Naßauskiesungs-Beschluß enthält, hinzu, ergibr sich, daß das Bundesverlassungsgericht die legislativen Entscheidungsräume weitgchend offon gehalten und sich selbst die Basisvoraussetzungen für eine Intervention in den umweltpolirischen Prozeß geschaffen hac. Dies ging zwar im Endeffekt zunächst meistens zu Lasten der umwcleschutzorientierten Interessen, zeigi jedoch auch, daß aktuell noch der Geserzgeber gefordert ist und auch gefordert werden kann. Weder reicht der Hinweis aul die Bestandsgarantic des Art. ${ }_{14}$ GG aus, um der Umweltpolitik restrikuve Grenzen zu serzen, noch ergibr sich bisher aus Art. 2 II GG ein einklagbarer Anspruch auf eine Geserzgebung, in der die ökologischen Anliegen den unumschränkcen Vorrang beanspruchen könnten. Insoweit also ergebnisbezogen offen, wird sich der verfassungsrechtliche Prüfungsrahmen - wenn überhaupr - auf die form- und funkcionsgerechre Entwicklung von Umweltschurzprogrammen beziehen müssen.

Dies bedeutct zunächst, daß sich die Rechemäßigkeitskontrollen niche nur auf die Prüfung des Zustandekommens, sondern vor allem auf die Steuerungs- und Entwicklungsfähigkeir nachfolgender Programmentwicklungsphasen bezichen sollsen. In diesem Sinne härte die Rechrsprechung zu allererst dafür zu sorgen, daß die auch in der Umwelepolitik dominierende Neigung zu dilatorischen Formelkompromissen dic Gesetzgebung nicht voilends in rituellen Übungen rhetorischer Problembearbeitungen auflöst. Die auch für das Umweltrecht zu beachtende w Wesentlichkeitscheorie "könnte dabei durch eine prozeßorientierte Interpretation problemadäquate Kontrolldimensionen eröffnen ${ }^{81}$. Hier dürften umfangreiche Aufgaben für eine kritische Durchforstung der bisherigen Umweltgeserzgebung auf prozedurale Mängel zu bervältigen sein, die letztlich eine konzeptionelle Neuordnung der Gesetzgebung erfordern

81 V V J dazu Wolf (FN 21), S. 452 I. 